\title{
Perceptions and Experiences of Healthcare Personnel Regarding Violence Against Health Care: A Qualitative Multiple Case Study
}

Muhammad Naseem Khan ( $\nabla$ drnasim@hsa.edu.pk)

Health Services Academy

\section{Babar Awan}

Khyber Medical University

\section{Mirwais Khan}

International Committee of the Red Cross

\section{Usman Mehboob}

Khyber Medical University

\section{Zia Ul Haq}

Khyber Medical University

Naeem Ullah

Saidu Medical College

Mohsin Zohaib

Khyber Medical University

Faryal Baddia

International Committee of the Red Cross

\section{Salman Khan}

International Committee of the Red Cross

\section{Maciej Polkowski}

International Committee of the Red Cross

\section{Research Article}

Keywords: Workplace violence, violence against health care, qualitative study.

Posted Date: February 18th, 2022

DOI: https://doi.org/10.21203/rs.3.rs-273638/v2

License: (1) (1) This work is licensed under a Creative Commons Attribution 4.0 International License.

Read Full License 


\section{Abstract}

\section{Background}

Healthcare workers are at high risk of facing violence all over the world. The sanctity, respect,and protection of health care beingthreatened by violence in healthcare settings. The study aims to explore the perception of healthcare personnel about violence in healthcare settings in district Peshawar.

\section{Methods}

This qualitative multiple case study uses a participatory approach to understandthe processes and themes that explain the healthcare personnel's experience of violence. Ten focus group discussions and three individual in-depth interviews were conducted with eighty-three participants recruited through purposive sampling.

\section{Results}

Three distinct recurrent themes emerged from the responses of the different stakeholders, and a consensus was reached on seven broad categories. The themes were: (a) Not all the wounds are visible: the theme describes the nature, frequency, and characteristics of violence and explores that violence is not merely any physical action taken against men or women to cause visible physical wounds, but also includes verbal or emotional abuse that attempts to hurt the feelings or affects the health of an individual. (b) But violence is never the answer: different categories, including the perpetrators of violence, causes, and contributing factors, and the consequences and effects of violence on individuals and institutions were explored and summarized that there could be many causes to provoke violence, but still violence cannot be justified. (c) Vaccine for violence: describesthe various strategies for the prevention of violence and suggests multiplemeasures at the public and institutional levels to be incorporated for a future without violence.

\section{Conclusion}

The study concluded that violence against healthcare is a frequently occurring phenomenon inhealthcare settings. Most of the incidents are not highlighted as they are verbal/emotional but have far more impact on the healthcare personnel. A holistic approach was suggested for ending violence in the healthcare settings, andthe need to involve all relevant stakeholders was emphasized. The recommended measures include policy formulation, promoting awareness and education, capacity building of healthcare personnel on communication strategies/consultation skills, improved security of the healthcare settings,and positive role of media in promoting the respect of healthcare personnel. Further research should then focus on evaluating the effectiveness of these measures.

\section{Background}


Globally, violence against healthcare providers is a persistent public health problem that is ubiquitous, under-reported, and largely ignored (1). According to the World Health Organization (WHO), violence is defined as "the intentional use of physical force or power, threatened or actual, against oneself, another person, or against a group or community that either result in or has a high likelihood of resulting in injury, death, psychological harm, mal-development or deprivation" $(2,3)$. It has been shown by many research studies that incidents of violence occur frequently and repeatedly in healthcare settings (4). The prevalence of violence in healthcare settings is more significant than in other occupational settings, and healthcare workers have a five times greater risk of being the victims of violence compared to workers in all other industries combined $(1,5)$. In the healthcare settings, it affects people belonging to all professional categories, including doctors, nurses, paramedics, and others (4) in all working environments, but the most frequent victims are those working in high-risk and stressful settings such as the emergency department and the psychiatry wards $(4,5,6)$. Various forms of violence, including physical and non-physical violence, are common in the industrialized as well as in the developing and transitional countries (7).

According to a report released by ILO/ICN/WHO/PSI, violence in healthcare settings is a universal issue causing psychological and physical problems, and its prevalence varies by country (8). Violence in the healthcare settings causes serious health, safety, and legal consequences (9). The victims suffer from the feelings of being left on their own by the institutions; scared, hurt, and angry (10). A cross-sectional survey was conducted in Chinese hospitals to determine the prevalence of workplace violence against nurses. A total of $71.9 \%$ of the nurses reported non-physical violent experiences, and $7.8 \%$ reported physically violent incidents in a year. Perpetrators were patients or their relatives $(93.5 \%$ and $82 \%$ respectively) (8). A nationwide study in the major tertiary care hospitals in Pakistan found more than $70 \%$ of healthcare personnel facing violence in the emergency departments during the preceding two months of the survey. Verbal abuse was $65 \%$, while physical abuse was faced by around $12 \%$ of the emergency physicians (11). Similarly, a study in Karachi, Pakistan, reported one in six and three in five physicians reported physical or verbal abuse, respectively, in the past 12 months (5).

Pakistan has a mixed health system with the involvement of the public, private, parastatal, civil societies, philanthropic organizations, and donor agencies. The public health delivery system functions through four modes of preventive, promotive, curative, and rehabilitative services spread across the country in the shape of primary, secondary, and tertiary health care facilities. Promotive and preventive services are mainly in the domain of the primary healthcare facilities, while the curative and rehabilitative services are focused in the secondary and tertiary healthcare facilities (12). Most of the secondary and tertiary health care facilities are overburdened as patients attend on their own with no proper reference system from the underutilized primary health care in the country (12). As a result, the hospitals are exhausted with limited control over the patients and their attendants.

Peshawar is the capital, and the most populace district of the province was chosen for the current study as most of the hospitals are tertiary level, with patients coming from all over the province. A recent survey conducted in Peshawar (current study site) found that around half of the healthcare workers have 
experienced physical and/or verbal violence in the past year, reflecting the high burden of this critical public health issue (13). There is limited literature and no qualitative research on the current topic in the local settings. Therefore the present study was aimed to explore the perceptions of healthcare personnel about violence in the healthcare settings in district Peshawar. This is further broken down into factors leading to the incidence of violent events, perpetrators of the violence, consequences of the violence on the victims and the existing social or legal services, and the recommendations for the prevention of such incidents in the future.

\section{Methods}

\section{Study Design}

This qualitative research used multiple case study approach (14) to explore the perceptions of different stakeholders, including doctors, nurses, support staff (ward orderlies, ambulance drivers, and gatekeepers), administration (Deputy Medical Superintendent), physiotherapists, media personnel, and police regarding violence against healthcare personnel in district Peshawar, Khyber Pakhtunkhwa, Pakistan. The healthcare personnel was invited mainly from the three tertiary care public sector hospitals of district Peshawar. Apart from these physiotherapists working at a teaching institute, and police and media personnel were also invited to participate.

\section{Sampling}

For the FGDs, participants were selected through the purposive sampling technique. Healthcare workers who had experienced and/or witnessed violence in the past year were invited from the three leading tertiary care hospitals and the physiotherapy institute in the district. In comparison, the three in-depth interviews were conducted purposively with the individuals involved in some capacity with the violent incidents in the hospital administration, police, and media. A total of ten Focus Group Discussions (FGDs) and three individual in-depth interviews (police, hospital administration, and media) were conducted. The minimum number of participants was five and a maximum of twelve in each FGD.

\section{Data Collection}

Among the FGDs, three FGDs, each with doctors, nurses, and support staff, were conducted in each of the three leading tertiary care hospitals. One FGD was conducted with the physiotherapists from the teaching institute. The number of participants in the FGDs ranged between six to twelve. Each of the FGD, on average, lasted for around 60 minutes. Three individual in-depth interviews were conducted onsite; one with the deputy medical superintendent (administration), another with police representative of the hospital, and third with media personnel. The in-depth interviews were completed in around 40 minutes. The FGDs and interviews were audio-recorded. At the start, the participants were apprised of the purpose of the study, and their informed consent was obtained. Ethical approval was obtained from the Ethics 
Review Board of Khyber Medical University. Permission was also obtained from the respective institution where the FGDs and in-depth interviews were conducted. At the start of the interview, the moderator assured the respondent about confidentiality. Open-ended questions were used to gather data from individual participants and during FGDs. Guiding questions were searched from literature and further authenticated by three experts in the field of qualitative research at Khyber Medical University. A discussion was generated about the participant's perspective of violence against healthcare personnel, the context of workplace violence, causes of violence, institutional response, and suggestions/recommendations to prevent the incidents of violence. All the responses were kept confidential and anonymous. All the FGDs and in-depth interviews were conducted by the same moderator.

\section{Data Analysis}

Transcriber software was used for the transcription of data. The first cycle of coding or initial analysis was done by open coding. The second cycle of coding was done to find out relationships by axial coding and to develop categories and themes. Then, thematic analysis was done to create meaningful patterns. Thematic analysis was performed through the process of coding in six phases to create meaningful patterns. These phases were: familiarization with data, generating initial codes, searching for themes among codes, reviewing themes, defining and naming themes, and producing the final report $(15,16)$. The first four authors were engaged in the data analysis and initially developed a consensus amongst themselves, followed by review, discussion, and agreement with authors 5 to 9 over online group meetings.

\section{Results}

A total of fifty-two codes were extracted from the transcripts that were categorized under seven broad categories. The seven broad categories led to three distinct themes under which we reorganized twelve subthemes. We organized five subthemes under the first theme, three subthemes under the second theme, and four subthemes under the third theme.

The three themes were;

1. Not all the wounds are visible

2. But violence is never the answer

3. Vaccine for violence

\section{Theme I:}

\section{Not all the wounds are visible:}


The first theme that emerged from this study metaphorically describes that violence is not merely any physical action taken against men or women to hurt them or cause visible physical wounds, but it includes verbal or emotional abuse of any form that directly or indirectly attempts to hurt the feelings or affects the health of an individual. This is the most prevalent and under-reported, and unnoticed form of violence. Five sub-themes were identified for the theme-l, and a consensus was reached on two broad categories: description of violence and prevailing status of violence (including experiences of workplace violence and nature, characteristics, and frequency of violence). The subthemes were:

\section{Perceived personalization of violence}

The narrative illustrates the meaning that the healthcare providers ascribed to violence. The violence that was directed towards healthcare providers as representative of the 'system' had a less negative impact on their emotional wellbeing as compared to violence perceived as 'personal'. Participants reported feelings of emotional distress by the acts of violence which they perceived as directed to their 'person'. One of the representative quotes was:

"Once a patient's attendant ran to hit me because his patient died of an end-stage organ disease that cannot be cured, he started yelling at me and was staring aggressively" (FGD Doctors-I)

2. Verbal abuse as a weapon of choice

Findings indicated that the healthcare staff experienced frequent verbal abuse at the hands of patients and their relatives. Verbal abuse was an almost daily occurrence and appeared as the first choice of violence by the perpetrators. One of the representative quotes was:

"Most of the time when the patients' attendants are told to stay out of ER/ward, they start misbehaving, yelling/ staring aggressively". (FGD Nurses-I)

3. Victimization of healthcare personnel

The narrative illustrates that healthcare personnel was being victimized easily in different ways, such as threats, physical assaults, and verbal abuse by the perpetrators. One of the representative quotes was:

"Once a patient's attendants came with guns, and they wanted to fight with CMO (Casualty Medical Officer) on duty because CMO told them to wait outside the ER." (Interview Administrator)

\section{Psychological/emotional abuse: a hidden form of violence}

Psychological or emotional distress was identified as lethal as physical violence by the participants. This is a hidden form of violence and, most of the time affects the healthcare workers as a consequence of physical and verbal violence. This is not the apparent form of violence but is as lethal as physical violence. One of the representative quotes was: 
"I have observed the incidents of emotional abuse and verbal threats against workers at or outside the hospital." (Interview Media)

5. No excuse for physical abuse

This subtheme described that physical abuse could not be justified on any ground. There should be no room for any form of violence; however, physical abuse is not justifiable, and it should be legally addressed. One of the representative quotes was:

"Workplace violence is an occupational safety and health hazard. It produces a bad impact on individuals' mental health and institutions' integrity." (Interview Media)

\section{Theme II:}

\section{But violence is never the answer:}

The second theme of the qualitative study explored that there can be many causes to provoke violence, but still, violence cannot be justified. Through this theme, the different causes of violence at the institutional, public, and government levels are described in Table 1. At the healthcare institutional level, the causes circle around lack of policy/security, overburdened health facilities leading to inadequate and poor quality services, and desensitization due to the prevalent nature of violence in healthcare institutions. At the general level, the causes of violence are illiteracy, poverty, intolerance, long waiting times (overburdened health facilities as described above), and negative image through social media. At the governmental level, the causes of violence in the healthcare institutions are lack of implementation of security act, political interference, top-bottom approach, underutilized primary healthcare facilities and nonexistent referral mechanisms, defamation of healthcare institutes, and lack of population control.

Three sub-themes were identified for theme II, and a consensus was reached on three broad categories, i.e., perpetrators of violence, causes, and contributing factors, and effects of violence on individuals and institutions. The sub-themes identified:

1. Healthcare institutions are no longer 'safe havens'.

The participants showed their concern that because of the everyday occurrence of violence in healthcare institutions, the institutions had lost their identity as a place of protection. The safety of the healthcare institution is in danger not only from the outside (patients and attendants) but also from the inside. The female healthcare personnel is harassed by the dominant male colleagues. One of the representative quotes was:

"Patients/attendants create problems on a daily basis. Ward orderlies and admin staff are also responsible. They are involved in harassment (mostly psychological)." (FGD Nurses-II)

2. Violence is the problem, not the solution 
This sub-theme identified numerous causes of violence and illustrated that despite all reasons, violence initiated by the perpetrators was not the solution. This is because most of the perpetrators are illiterate or politically spoiled and just look for excuses to opt for violence. One of the representative quotes was:

"In my opinion, patients' companion, illiterate and those who have political background come to the hospital with a traditional mindset. They look for excuses for creating violence." (Interview Police)

\section{A fear that's palpable}

This sub-theme illustrated that the emerging incidence of violence is notably fearful and emphasized the different causes of violence that can be addressed at the government level.

Table 1

Causes and contributing factors of violence

\section{Healthcare Institution: No longer safe havens}

(At institution level)

Policy vacuum

Inadequate management/administration support

Heavy workload

Improper service delivery

Lack of cooperation/collaboration

Lack of proper security system

Deficiency of Human resources and facilities

Chaotic workplace environment

Desensitization or habituation

Workplace negligence

\section{Violence is the problem, not the solution}

(At public level)

education

Delayed attention/long waiting time

Unreasonable expectations due to government claims

Situational trigger

- Impatience and intolerance

Increase poverty and unemployment

A high rate of inflation

Role of social media
A fear that's palpable

(At government level)

Lack of implementation of security act/policy

Culture of political intrusion

Culture of intercession

Top-bottom cultural assumption

Defamation of healthcare institutes

- High economic costs

- Underutilized primary healthcare facilities

Lack of a proper referral system Lack of proper monitoring

Lack of population control Lack of assessment of data for planning and development

\section{Theme III:}

\section{Vaccine for violence:}

The third theme that emerged describes the various strategies for the prevention of violence (Table 2). The primary vaccine (prevention) for violence is suggested to be at the governmental level, and that is to 
increase literacy, employment, advocacy/awareness, decrease poverty, and better policy and legislation. At the institutional level, more focus should be given to the security of the hospital and better quality services. For those events that could not be prevented, training of the staff on de-escalation of violence, situational analysis/problem solving, and better communications are the need of the day. Lastly, the establishment of a rapid response system to react to events of violence and counseling and rehabilitation centers for the affected.

Four sub-themes were identified for theme-III, and a consensus was reached on two broad categories, that is, institutional response and prevention of violence. The sub-themes identified were: future without violence, the establishment of institutional regime/model for a safe working environment, strategies for trigger phase prevention, and strategies for crisis de-escalation and protection.

\section{Future without violence}

The study participants wished for a future without violence against healthcare workers. The representative quote for this sub-theme was:

"People should be educated through awareness campaigns regarding avoiding violence. Media should play a positive role in portraying doctors' image." (FGD Doctors-III)

2. Establishment of institutional regime/model for a safe working environment

The study participants, especially the females, also proposed a safe institutional environment. The representative quote for this sub-theme was:

"People should be made aware of the punishment for violence at the hospital. There should be the proper security system." (FGD Nurses-II)

3. Strategies for trigger phase prevention

One of the study participants also suggested an approach for trigger phase prevention, such as;

"There should be a rapid emergency response system at all the hospitals." (Interview Administrator)

4. Strategies for crisis de-escalation and protection

Some of the participants also suggested crisis de-escalation and protection strategies such as;

"A culture of respect should be promoted at hospitals and government institutions. Coordination should be developed. (Interview Police)

"As we know that patients and their families are under stress, hospital staff should take good care of them. Furthermore, staff education, skills, security and health measures should be improved." (Interview Media) 
Table 2

Strategies for prevention of violence

\begin{tabular}{|c|c|c|c|}
\hline $\begin{array}{l}\text { (Primary prevention at } \\
\text { government level) }\end{array}$ & $\begin{array}{l}\text { Establishment of } \\
\text { institutional regime/model } \\
\text { for a safe working } \\
\text { environment } \\
\text { (Primary prevention at } \\
\text { institution level) }\end{array}$ & $\begin{array}{l}\text { Strategies for } \\
\text { trigger phase } \\
\text { prevention } \\
\text { (Secondary } \\
\text { prevention) }\end{array}$ & $\begin{array}{l}\text { Strategies for } \\
\text { crisis de- } \\
\text { escalation and } \\
\text { protection } \\
\text { (Tertiary } \\
\text { Prevention) }\end{array}$ \\
\hline $\begin{array}{l}\text { Education } \\
\text { Peace education } \\
\text { Advocacy/awareness/outreach } \\
\text { A collaboration of all } \\
\text { stakeholders/departments } \\
\text { Influencing policy and } \\
\text { legislation } \\
\text { Increase in employment } \\
\text { opportunities } \\
\text { Decrease in poverty } \\
\text { The positive role of social } \\
\text { media }\end{array}$ & $\begin{array}{l}\text { Droper service delivery } \\
\text { n Implementation of } \\
\text { security act (making } \\
\text { healthcare institutions as } \\
\text { "zero-tolerance for } \\
\text { violence" zones) } \\
\text { Establishment of } 24 \\
\text { hours security surveillance } \\
\text { system } \\
\text { Good environmental } \\
\text { policy } \\
\text { collegial awareness } \\
\text { averting violence } \\
\text { Increase human } \\
\text { resource } \\
\text { Increase institutional } \\
\text { capacity } \\
\text { Staff education, } \\
\text { training, and skill } \\
\text { development }\end{array}$ & $\begin{array}{l}\text { Empathizing } \\
\text { with patients and } \\
\text { their } \\
\text { attendants/family } \\
\text { Conveying } \\
\text { caring } \\
\text { G Fostering } \\
\text { resilience } \\
\text { Situation } \\
\text { analysis and } \\
\text { problem-solving } \\
\text { of Development } \\
\text { of good } \\
\text { communication } \\
\text { skills }\end{array}$ & $\begin{array}{l}\text { Establishment } \\
\text { of rapid and } \\
\text { effective } \\
\text { emergency } \\
\text { response } \\
\text { system } \\
\text { (REERS) } \\
\text { Establishment } \\
\text { of counseling } \\
\text { and } \\
\text { rehabilitation } \\
\text { center } \\
\\
\text { Development } \\
\text { of } \\
\text { compensation } \\
\text { system }\end{array}$ \\
\hline
\end{tabular}

\section{Discussion}

This study contributes knowledge regarding the issue of violence against healthcare staff, particularly in district Peshawar, Khyber Pakhtunkhwa, where little qualitative research on this subject has been done previously. This study aimed to explore the views of hospital stakeholders regarding incidents of violence. The three main themes that emerged from the qualitative analysis reflected the stakeholders' overall view of workplace violence as a health and safety issue for the hospital employees.

Most of the stakeholders described the violence as any form of verbal, physical, and psychological abuse. The first theme reflected that not all the wounds are visible and reiterated that most of these violent events are not reported and noticed in the hospital settings and are considered as routine events with the job. Almost all the stakeholders regarded verbal abuse as a weapon of choice and the most common form of violence. Incidents of verbal, emotional, and psychological violence are not reported and 
are far more common in comparison with physical violence. This finding is supported by literature from national and international settings where verbal abuse was said to be the predominant form of violence $(13,17-19)$. This does not mean that physical violence is not a problem in hospitals settings and does not require any attention. Physical violence is recently reported in the local healthcare settings of Peshawar (13) and Karachi (20) in Pakistan. On the contrary, there should be no room for physical violence in hospital settings and could not be justified on any ground.

Additionally, the healthcare professionals were finding themselves as a victim of violence. They described that such situations were unavoidable, and most of the time, they felt scared and emotionally drained. They perceived emotional or psychological abuse as a hidden form of violence being practiced routinely and highlighted that personal violence is more damaging than the one targeted against the system. Similar findings were reported in a review, where it was stated that reported incidents of violence represent only the tip of the iceberg, being under-reported because of the perception that it comes with the job. The resulting consequences to the victim included psychological burnout (21).

The second theme elaborated that there could be many reasons and causes of violence in the healthcare settings, but it is never the answer. The healthcare professionals shared their negative experiences regarding the behavior of people towards them. They believed most of the time people wanted selfdirected care, and any disagreement with them provides an excuse for reverting violence. Low literacy, overburdened health facilities leading to delayed attention, and long waiting times are some of the reasons at the public level. Lack of education based on the views of the participants is the most important cause of violence in healthcare settings. This view is supported by literature from the local settings where illiteracy and lack of awareness among the public were considered as an important cause of violence (13). Furthermore, the lack of implementation of security acts and political involvement were found to be the most important reasons for violence that should be addressed at the level of government. These findings are consistent with previous studies $(13,22-23)$. Most of the victims of physical abuse were those who tried to stop the perpetrators. This issue was highlighted in a cross-sectional study from Iran, where it was reported that most of the victims of physical violence were those who were trying to stop the aggressors and lack of people's knowledge about employees' tasks was the contributing factor (24).

Different stakeholders were of different opinions regarding the causes and contributing factors of violence, such as, "there are many causes that provoke violence such as lack of facilities or malfunctioning of equipment, long waiting times, intolerance or illiteracy of the public, but does that give anyone the right to abuse the service provider?". Moreover, they believed that policy vacuum, inadequate management of administration, and lack of collaboration and security system at institutes create a chaotic workplace environment that results in improper service delivery and frequent incidents of violence. Staff were desensitized to incidents of violence and considered them as a part of their daily routine. The healthcare workers desensitize due to the daily nature of these incidents, as reported in a mixed-method study from Gambia (22). 
The current study also found that such incidents of violence harmed individuals ' health and institutions. Victims passed through psychological stress and remained silent most of the time. They described that such incidents negatively affected their lives and patient care. To further add to their anxiety, there was a lack of coping mechanisms at the institutions. Similar findings were also reported that there is a relationship between stress and workplace violence. The tension generated due to violence can take the most extreme forms, such as burnout or suicide (25).

The participants reported that most of the time, patients' attendants, family members, or sometimes the patients themselves were the perpetrators of violence. They were directly involved in such incidents. These people were either uneducated or politically spoiled. However, in some situations, the colleagues, hospital staff, and the administration were indirectly involved by practicing personal, psychological, or power harassment. This kind of violence, as reported, is another significant area needing further exploration in future research. Such findings are reported in the literature in other countries as well $(13$, 26).

The third important theme described various strategies for the prevention of violence at the governmental and institute levels. Among the many prevention strategies, promoting education is the key to prevent violence as reported by the participants. Similarly, creating awareness among the public regarding avoidance of violence can make a big difference. Advocacy for violence prevention and implementation of policies regarding the security of healthcare institutes were found to be essential steps towards a safe working environment. Making hospitals "zero-tolerance zones"for violence was identified as a necessary factor in averting violence. The importance of education and violence prevention programs for a safe working environment is supported through literature (27).

In the present study, it was found that staff education, collaboration, and skill development were necessary for conveying care and averting violence. Good communication skills and fostering resilience was found essential for preventing and handling situational triggers. De-escalation training of hospital staff has shown promising results in the local context $(28,29)$. Facilitating hospital processes and managing attendants were identified as essential factors in preventing violence (30).

Our study findings also suggested the establishment of effective emergency response and security system for crisis de-escalation and protection. The participants said that there should be a 24-hours security surveillance system including scanners, cameras, security surveillance officers, and a security center at all hospitals. It was also identified that there should be a rehabilitation center and the development of a compensation system for the victims of violence, which is consistent with the results of a previous study (31).

Our study has holistically addressed the issue of violence against healthcare workers from a conservative culture. Some of the reasons for violence against healthcare workers have already been addressed previously from other cultures. Our study further adds to the existing literature and also provides a structured approach to avoid violence against healthcare workers. 
The study is limited in that it did not include the experiences of healthcare staff in small and remote areas' health facilities. The study did not have the perspectives of patients and their attendants. The findings also did not thoroughly differentiate the risks for violence by specific occupation or type of facility. Despite these limitations, the study was informative. It revealed that, in the experience of healthcare workers interviewed, violence in the Khyber Pakhtunkhwa healthcare system has become normalized or as an unavoidable risk.

\section{Conclusion}

Results of the study indicate the need to recognize that workplace violent events are observed frequently. The causes and contributing factors for violence against healthcare personnel have been documented in this study, and many solutions have been put forward. Ending violence against healthcare staff is dependent on efforts from various levels. National strategies should be developed to respond to violence against healthcare personnel. Stakeholders should be widely and comprehensively consulted about their views and ideas. The recommended measures include policy change, promoting education and awareness, training of healthcare staff, improved security, promoting the role of media to respect and reward the image of healthcare staff, and increasing the institutional capacity. Further research should focus on evaluating the effectiveness of these measures.

\section{Abbreviations}

CMO Casualty Medical Officer

ER Emergency Room

FGD Focus Group Discussion

ICRC International Committee of the Red Cross

REERS Rapid and Effective Emergency Response System

WHO World Health Organization

\section{Declarations}

\section{Ethics approval and consent to participate}

The study was approved by the Ethics Review Board of Khyber Medical University, Peshawar. Informed written consent was obtained from study participants following an explanation of the study aims and objectives. All procedures were performed with strict ethical standards.

\section{Consent for publication}


$\mathrm{N} / \mathrm{A}$

\section{Availability of data and materials}

The interview data generated and analyzed during the current study are not publicly available to protect patient confidentiality but are available from the corresponding author on reasonable request.

\section{Competing interests}

The authors declare that they have no competing interests.

\section{Funding}

This study was conducted under the framework of the Health Care in Danger (HCiD) initiative of the International Committee of the Red Cross (ICRC). The funding body has no role in the design of the study and collection, analysis, and interpretation of the data and writing of the manuscript.

\section{Authors Contributions}

MNK, BA, ZUH, MK, and MP conceived the idea of this research study and participated in its coordination. $\mathrm{BA}, \mathrm{NU}, \mathrm{MZ}, \mathrm{FB}$, and SK conducted field research activities and were involved in the data collection, cleaning, and initial analysis. Further analysis was supported by MNK, UM, BA, and MK. All authors contributed towards preparing the first draft as well as editing and reviewing the subsequent drafts. The authors read and approved the final manuscript.

\section{Acknowledgments}

The authors gratefully acknowledge the numerous study participants who made themselves available for interviews and focus group discussions. We are also very thankful to the healthcare administration. We are highly grateful to the management of the International Committee of the Red Cross, Pakistan, for the technical and financial support of the study.

\section{References}

1. Brophy JT, Keith MM, Hurley M. Assaulted and unheard: violence against healthcare staff. New solutions: a journal of environmental and occupational health policy. 2018;27(4):581-606.

2. WHO | World report on violence and health [Internet]. WHO. World Health Organization; [cited 2020 Oct 23]. Available from: https://www.who.int/violence_injury_prevention/violence/world_report/en/

3. WHO | Definition and typology of violence [Internet]. WHO. World Health Organization; [cited 2020 Oct 23]. Available from: https://www.who.int/violenceprevention/approach/definition/en/

4. Magnavita N. Workplace violence and occupational stress in healthcare workers: A chicken-and-egg situation-results of a 6-year follow-up study. Journal of nursing scholarship. 2014;46(5):366-76. 
5. Zafar W, Siddiqui E, Ejaz K, Shehzad MU, Khan UR, Jamali S, et al. Health care personnel and workplace violence in the emergency departments of a volatile metropolis: results from Karachi, Pakistan. The Journal of emergency medicine. 2013;45(5):761-72.

6. Lepping P, Lanka SV, Turner J, Stanaway SE, Krishna M. Percentage prevalence of patient and visitor violence against staff in high-risk UK medical wards. Clinical medicine. 2013;13(6):543.

7. Nelson R. Tackling violence against healthcare workers. The Lancet. 2014;383(9926):1373-4.

8. Jiao M, Ning N, Li Y, Gao L, Cui Y, Sun H, et al. Workplace violence against nurses in Chinese hospitals: a cross-sectional survey. BMJ open. 2015;5(3).

9. Hassankhani H, Parizad N, Gacki-Smith J, Rahmani A, Mohammadi E. The consequences of violence against nurses working in the emergency department: A qualitative study. International emergency nursing. 2018;39:20-5.

10. Ramacciati N, Ceccagnoli A, Addey B. Violence against nurses in the triage area: An Italian qualitative study. International emergency nursing. 2015;23(4):274-80.

11. Mirza NM, Amjad Al, Bhatti ABH, Shaikh KS, Kiani J, Yusuf MM, et al. violence and abuse faced by junior physicians in the emergency department from patients and their caretakers: a nationwide study from Pakistan. The Journal of emergency medicine. 2012;42(6):727-33.

12. Kumar S, Bano S. Comparison and analysis of health care delivery systems: Pakistan versus Bangladesh. J Hosp Med Manage. 2017;3(1):21-2.

13. Khan MN, Haq ZU, Khan M, Wali S, Baddia F, Rasul S, Khan S, Polkowski M, Ramirez-Mendoza JY. Prevalence and determinants of violence against health care in the metropolitan city of Peshawar: a cross sectional study. BMC public health. 2021 Dec;21(1):1-1.

14. Merriam SB. Case studies as qualitative research. Qualitative research and case study applications in education. 1998:26-43.

15. Lincoln YS, Guba EG. Naturalistic inquiry (vol. 75). Sage Thousand Oaks, CA; 1985.

16. Nowell LS, Norris JM, White DE, Moules NJ. Thematic analysis: Striving to meet the trustworthiness criteria. International journal of qualitative methods. 2017;16(1):1609406917733847.

17. Talas MS, Kocaöz S, Akgüç S. A survey of violence against staff working in the emergency department in Ankara, Turkey. Asian nursing research. 2011;5(4):197-203.

18. Abed M, Morris E, Sobers-Grannum N. Workplace violence against medical staff in healthcare facilities in Barbados. Occupational medicine. 2016;66(7):580-3.

19. Franz S, Zeh A, Schablon A, Kuhnert S, Nienhaus A. Aggression and violence against health care workers in Germany-a cross sectional retrospective survey. BMC health services research. 2010;10(1):1-8.

20. Baig LA, Shaikh S, Polkowski M, Ali SK, Jamali S, Mazharullah L, et al. violence against health care providers: a mixed-methods study from Karachi, Pakistan. The Journal of emergency medicine. 2018;54(4):558-66. 
21. Dubb SS. It doesn't" come with the job": violence against doctors at work must stop. BMJ: British Medical Journal (Online). 2015;350.

22. Sisawo EJ, Ouédraogo SYYA, Huang S-L. Workplace violence against nurses in the Gambia: mixed methods design. BMC health services research. 2017;17(1):1-11.

23. Hutchinson $M$, Jackson $D$. The construction and legitimation of workplace bullying in the public sector: insight into power dynamics and organisational failures in health and social care. Nursing inquiry. 2015;22(1):13-26.

24. Fallahi-Khoshknab M, Oskouie F, Najafi F, Ghazanfari N, Tamizi Z, Afshani S. Physical violence against health care workers: A nationwide study from Iran. Iranian journal of nursing and midwifery research. 2016;21(3):232.

25. Chappell D, Di Martino V. Violence at work: International Labour Organization. Asian-Pacific Newsletter on Occupational Health and Safety. 2006;6(1):2-9.

26. Park $\mathrm{M}$, Cho $\mathrm{S}-\mathrm{H}$, Hong $\mathrm{H}-\mathrm{J}$. Prevalence and perpetrators of workplace violence by nursing unit and the relationship between violence and the perceived work environment. Journal of nursing scholarship. 2015;47(1):87-95.

27. Gillespie GL, Gates DM, Miller M, Howard PK. Workplace violence in healthcare settings: risk factors and protective strategies. Rehabilitation nursing. 2010;35(5):177-84.

28. Baig L, Tanzil S, Shaikh S, Hashmi I, Khan MA, Polkowski M. Effectiveness of training on deescalation of violence and management of aggressive behavior faced by health care providers in a public sector hospital of Karachi. Pakistan journal of medical sciences. 2018;34(2):294.

29. Khan MN, Khan I, Ul-Haq Z, Khan M, Baddia F, Ahmad F, Khan S. Managing violence against healthcare personnel in the emergency settings of Pakistan: a mixed methods study. BMJ open. 2021 Jun 1;11(6):e044213.

30. Abdollahzadeh F, Asghari E, Ebrahimi H, Rahmani A, Vahidi M. How to prevent workplace incivility?: Nurses' perspective. Iranian journal of nursing and midwifery research. 2017;22(2):157.

31. Kamchuchat C, Chongsuvivatwong V, Oncheunjit S, Yip TW, Sangthong R. Workplace violence directed at nursing staff at a general hospital in southern Thailand. Journal of occupational health. 2008;50(2):201-7. 